\title{
Toggle toolkit: A tool for conducting experiments in unity virtual environments
}

\author{
Pavel Ugwitz ${ }^{1,2}$ (D) Alžběta Šašinková ${ }^{2}$ Čeněk Šašinka ${ }^{2} \cdot$ Zdeněk Stachoň $^{1,2} \cdot$ Vojtěch Juřík $^{2,3}$
}

Accepted: 9 November 2020 / Published online: 6 January 2021

(C) The Psychonomic Society, Inc. 2021

\begin{abstract}
This article presents and offers Toggle Toolkit, which is an original collection of Unity scripts designed to control various aspects of interactive 3D experiments. The toolkit enables researchers in different fields to design, conduct and evaluate experiments and include interactive elements in immersive virtual environments. This was achieved by using the internal functionalities of the Unity engine and solutions of our own design. The structure of Toggle Toolkit allows triggers and toggles to be allocated to existing virtual objects and throughout the Unity scene. Once a trigger is executed (with a pre-described action, such as colliding with a virtual object, pressing a key, gazing at an object, etc.), the toggles associated with the trigger are activated and then change the attributes or behaviors of linked objects. All interactive behavior is logged and made available for further statistical analysis. Examples of applications in research are presented and discussed. The Toggle Toolkit's utility lies in its simplicity and modularity. The Toolkit was especially produced for experimenters with few coding skills and high customization requirements in their experiments. The tool is freely available for use in research and can be enhanced with custom scripts. A video tutorial is provided to facilitate use of the tool. The paper aims to not only introduce beginners to experimentation with VR but also offers more experienced researchers who are potentially interested in using and adjusting the features the Toolkit a deeper insight into its structure.
\end{abstract}

Keywords Virtual environment · Virtual reality · Interactivity · Dynamic visualization · 3D visualization · Behavioral analysis . Experiment design $\cdot$ Event logger

Pavel Ugwitz

ugwitz.pavel@mail.muni.cz

$\triangle$ Alžběta Šašinková

st.betty@mail.muni.cz

Čeněk Šašinka

cenek.sasinka@mail.muni.cz

Zdeněk Stachoň

zstachon@geogr.muni.cz

Vojtěch Juřík

jurik.vojtech@gmail.com

1 Department of Geography, Faculty of Science, Masaryk University, Kotlářská 267/2, 61137 Brno, Czech Republic

2 Department of Information and Library Studies, Faculty of Arts, Masaryk University, Arna Nováka 1/1, 60200 Brno, Czech Republic

3 Centre for Experimental Psychology and Cognitive Sciences, Department of Psychology, Faculty of Arts, Masaryk University, Arna Nováka 1/1, 60200 Brno, Czech Republic

\section{Introduction}

The rapid development of technologies for virtual reality (VR), especially visual head-mounted displays, computer graphics, and general computing power (Singer, 2013; Cuervo et al., 2018), have paved the way for the creation of dynamic, controllable, replicable, customizable and complex stimuli that offer experiences which resemble real phenomena.

It is not a new phenomenon in science that experiments shift to the world of technology. In 1D experiments, many instruments have been successfully introduced and applied (e.g., Šašinka, Morong \& Stachoň, 2017; NeuroBS, 2017; Cornelissen, Peters \& Palmer, 2002; Paradigm, 2017; Mathôt, Schreij \& Theeuwes, 2012; etc.).

VR technologies have been suggested as stimulating brain mechanisms in a similar manner to real-world experiences (Alcañiz et al., 2009). The opportunity to explore human behavior and cognition in realistic, yet well-controlled experimental settings produced with VR represents an important improvement in research over the natural trade-off in science 
between experimental control and ecological validity, which has so far been difficult to overcome (Loomis et al., 1999).

So far, the necessity to possess programming skills has prevented many people from designing and conducting experiments in virtual environments unless they are members of profoundly transdisciplinary teams. This difficulty could be partly offset by deploying a visual scripting tool (Sewell, 2015; D., 2015), although if no scripts are available to base simplified scripting on, no solution is offered. Toggle Toolkit was developed for the needs of our own interdisciplinary research team in applying the Unity editor's user interface (Unity Inspector) to simplify experimental design, data collection and subsequent data analysis. Toggle Toolkit enables virtual users to interact with a virtual environment and the environment to react to a user. The toolkit also aids in tracking the behavior of virtual users, including their locomotion, gestures, manipulation of virtual objects, or even eye movements. Examples of the toolkit's use in producing a VR experiment are described in Chapter 3, Experimental Utility. To understand the workflow, see the linked tutorial video, which presents a simple example of an experiment managed using Toggle Toolkit: https://drive.google.com/file/d/ 1cMfmbaKH1pQfmNA3IW8BCSyhrXLTB-E9

Toggle Toolkit is based on a trigger-event concept extended by nested objects and states. The software design patterns that the kit is based on (command pattern, state pattern; Baron, 2019) have been applied in many $3 \mathrm{D}$ games and applications over the past 25 years. These functionalities, however, have not always been easily accessible to users because of limited functionality, previous-generation $3 \mathrm{D}$ engine architecture (e.g., Unreal Engine 2/3, Source, id Tech engines, and older), in-engine editors, the inability to customize closed-source solutions, or toolkits that did not match the needs of creating an experiment since they were only game-oriented.

Naturally, Toggle Toolkit is not the first ever software produced to enhance the process of designing experiments in VR. However, none of the other tools available to date are equivalent to Toggle Toolkit. EVE (Experiments in Virtual Environments) (Grübel et al., 2017) is a valuable tool which responds to similar research needs but approaches the problem from a different perspective. EVE differs from Toggle Toolkit in its range of functionality, its architecture and method of use. EVE includes inherent interaction, logging and evaluation scripts, and has utilities for physiological measurements, questionnaires and movement tracking. However, the tool's actual interactivity, as far as we understand from its GitHub repository $^{1}$, consists solely in concealing objects and displaying text. It is a type of "superstructure" laid over a finished and compiled Unity project, unlike Toggle Toolkit, which is applied during the process of creating a Unity project. Toggle Toolkit also contains a greater focus on detail in its modularity: the tool enables dependency chains to be created between individual

\footnotetext{
${ }^{1}$ https://github.com/cog-ethz/EVE/tree/master/Assets/EVE/Scripts
}

objects and non-trivial sequences of events. Unlike in EVE, every Unity object can become an interactive factor, and the variety of states any object can attain is limited only by its features. The EVE environment is likely easier to use for a common researcher (e.g., it does not require knowledge of Unity editor), however its adjustability compared to Toggle Toolkit is limited, which makes it less able to meet higher experimental design requirements.

Besides EVE, there are some partially analogous tools that possess similar functionalities but differ in other aspects. The reference Unity 2D Game Kit ("2D Game Kit" 2017) has its own interaction system for in-game objects that react to players. This game-centric solution is only partially customizable, but its trigger-event functionality follows the same principles as Toggle Toolkit. Although focusing primarily on VR controllers, VR UI, and small-scale object manipulation, the Unity XR Interaction Toolkit(Fuad, 2019) has some similarity in being able to alter virtual objects and the surrounding environment. The Unity Experiment Framework (Brookes et al., 2019) provides a comprehensive structure for behavioral experiments in virtual settings; direct access to Unity in-engine functionalities, however, is intentionally not offered and must be programmed manually. The Virtual Reality Experiments (VREX) Toolbox (Vasser et al., 2017) allows the creation of basic experiments situated in interior spaces that consist of a square grid of interconnected rooms generated in the researcher's user interface and detailed procedurally. While this is a useful function for some experiments, it is unnecessary to limit ourselves to this alone.

We chose to remain with Unity engine in our research, as Unity is relatively easy in programming functionality and has an existing support infrastructure (forums, tutorials, videos, documentation), component system (Unity Manual: Components, 2020), , accessibility through the generic C\# language and custom C\# extensions (Unity Scripting Reference, 2020), extension packages, versatility for use in different fields, and developer maintenance (as opposed to purely research-oriented 3D engines such as Vizard (Vizard, 2020) or older in-house implementations such as Vrecko (Kovalčík et al., 2012)).

Concerning published studies situated in virtual environments, use of multi-modal, complex dynamic implementations for research is currently highly unsatisfactory. Basic research is more prevalent, with designs that frequently rely on fully static, non-interactive environments or that merely implement a single function (e.g., user teleportation (Moghadam et al., 2018), object collection (Grübel et al., 2017), object manipulation (Caputo et al., 2018), movement measurements (Diersch, Wolbers, 2019), and animated passive viewing (Parong, Mayer, 2018)). In older experiments with closed-source 3D engines, the technical aspects of experimental procedures were not mentioned (Smith \& Trenholme, 2009; Gould et al., 2007). Assuming no automated in-engine interactivity or measuring 
took place in these studies, the analysis may have been transferred to a human researcher or post hoc protocol.

Toggle Toolkit requires few coding skills and offers a comprehensive solution that can be adjusted to suit the needs of various experimental designs and various technologies used (e.g. HMD, CAVE etc.). Compared to other similar tools and because of its modularity and focus on detail, Toggle Toolkit allows elements of very high interactivity and enhances a great degree of control in an experiment. The software is freely available and supplemented by an instructional video to provide information to less experienced VR researchers. The toolkit can significantly improve the effectiveness of an experiment's design process since it is based on the real needs of researchers.

The following chapter is especially interesting to researchers who have more advanced coding skills and whose aim is to gain insight into the toolkit's architecture and options for further development. Readers less interested in how the toolkit precisely functions may find the first section of this chapter sufficient and may skim through the Trigger and Toggle Script Module paragraphs to explore the figures and their descriptions or skip them entirely. Readers may then proceed to the Experiment Utilities section, which presents concrete examples of the toolkit's application in research.

\section{Software description}

Toggle Toolkit's main function is in predefining controlled changes encountered in a virtual environment through realtime $3 \mathrm{D}$ visualization experiments, i.e., altering the graphics and functionality and enabling dynamic visualizations and interactive elements. That is achieved by creating links between the Trigger and Toggle scripts included in the toolkit (for the principle of the toolkit's architecture, see Fig. 1). A stimulus activates a trigger script. This trigger script is linked to a toggle script appended to an object in the VR scene. Activating a trigger causes a linked toggle to change the state of its respective object. The change in the target object due to its associated toggle can be either instantaneous (e.g., a change of color) or continuous (e.g., an animation).

The following scenario (as depicted in Figs. 2 and 3) represents an example case of the aforementioned concepts: a user situated in a hotel backyard is instructed to enter the hotel. For clarity, the design process of this example is presented in the tutorial video mentioned above (https://drive.google.com/ file/d/1cMfmbaKH1pQfmNA3IW8BCSyhrXLTB-E9). The hotel door (i.e., object to be affected) is surrounded by an invisible collider (highlighted in Figs. 2 and 3 by a thin green line). Once the user touches the collider (i.e., activates the trigger), the hotel door opens (i.e., the game object toggles itself from closed to open). When the user leaves the area marked by the collider, the trigger is once again activated and the door returns to its original state (i.e., closes). This example illustrates a simple concept using Toggle Toolkit as an "on/off switch", where the game object has only two predefined states and repeated trigger activation leads to the object returning to its original state.

Toggle Toolkit components can be added to the objects or native components of a Unity scene and their values can be set up using the Unity editor through the Unity inspector GUI. Toggle Toolkit can thus access and alter Unity object attributes; while object attribute manipulation is native to the Unity editor inspector interface and Unity scripting, the tool permits unified, streamlined, continuous changes from a single interface, which is the toolkit's chief utility. When a scene setup and its desired Toggle Toolkit functionalities are finalized, the scene can be compiled and deployed for experimental purposes as a standalone application.

The toolkit was designed as a modular system: some of its components can reference other components, and the spectrum of application is broad. If additional components are

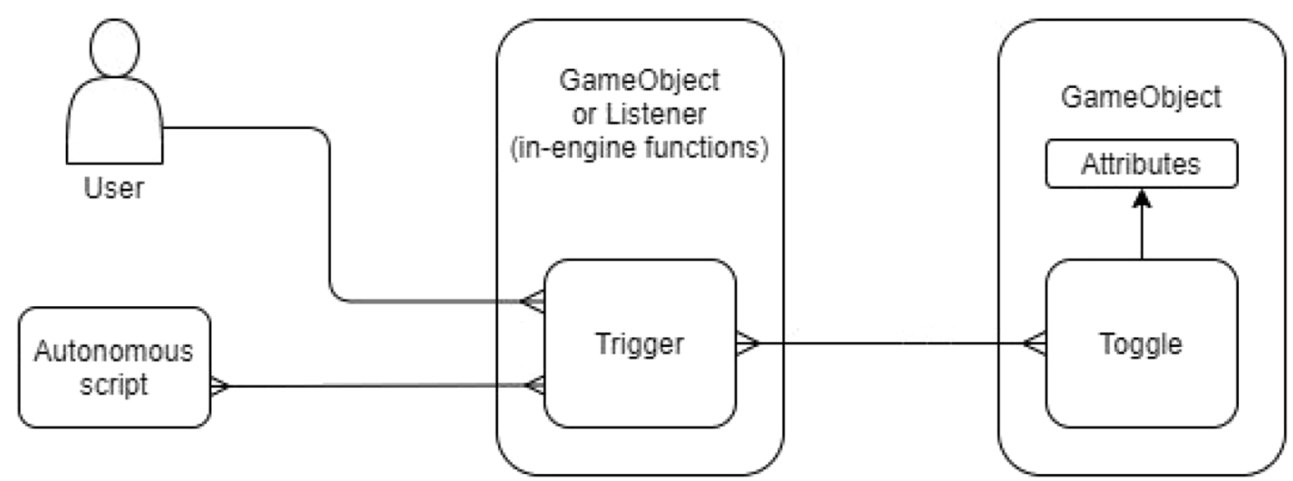

Fig. 1 Basic overview of Toggle Toolkit system architecture. Note. Usually, an external entity (a user or script) activates the trigger script(s), which, by extension, activates the toggle script(s) on associated object(s). When toggled, the object(s) change(s) their attributes, feeding back to the dynamics of the scene. This scheme implies that multiple objects can be interconnected, sometimes with dependencies or parent-object relationships. For further explanation of advanced concepts, see Chapter 2.2.2 


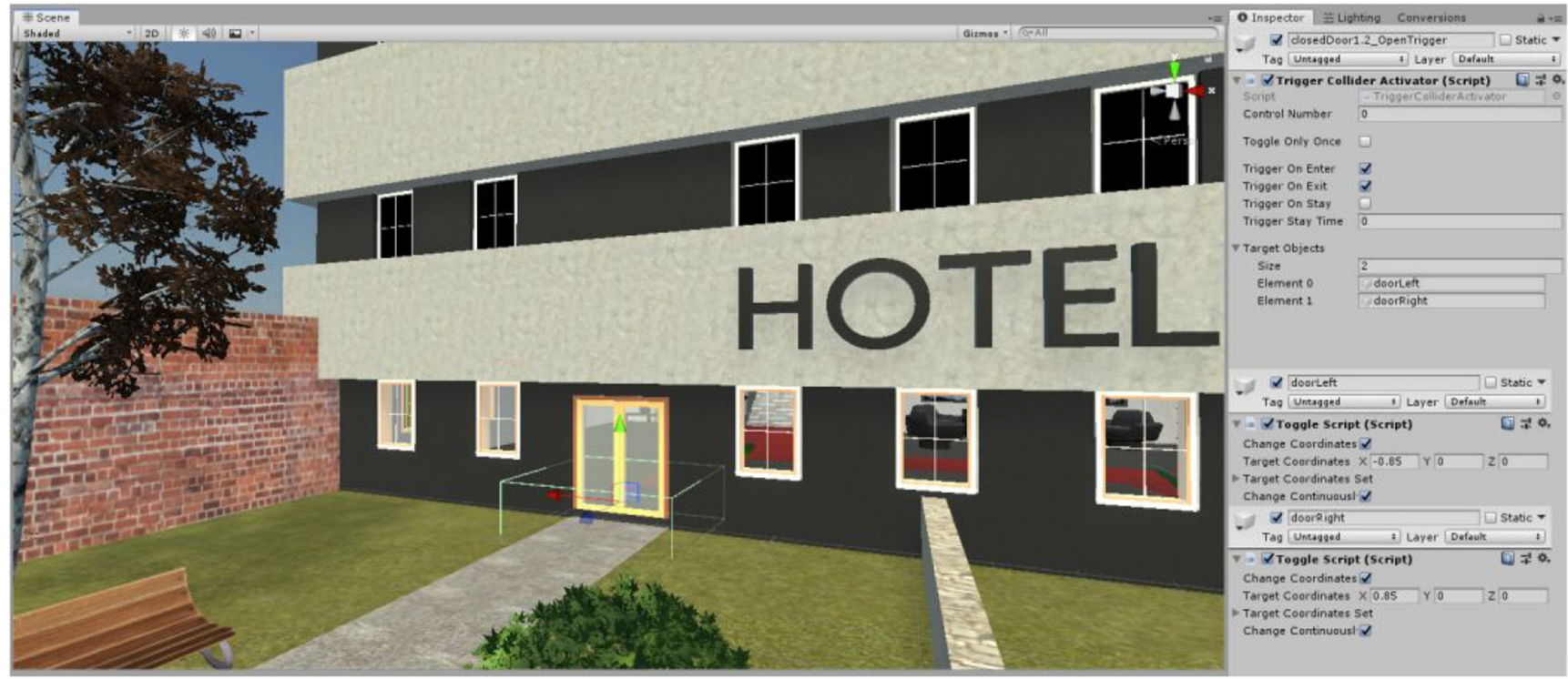

Fig. 2 Example of setting up a simple two-state (on/off) trigger. Note. A collider trigger is attached to the hotel door object; the trigger is set up to activate when an external entity (the user) collides with it and trigger again when the user no longer collides with it. The GameObjects associated with this trigger are the two door wings, and their toggle behavior

implemented, they can draw from the functionality of existing components (see chapter Discussion).

\section{Trigger script modules}

Trigger scripts have many variations, simply because each type of user controller interface or automated script that activates a trigger (Fig. 1) is unique and must be adapted according to its programming, controller logic or good HCI practices.

A trigger may be accessed from either the real, physical world (e.g., pressing a key on a keyboard or a controller button) or a virtual environment (e.g., stepping on a pressure board in a virtual room or passing through an automated revolving door in a virtual building, as illustrated in Figs. 2 and 3). moves the door wings $85 \mathrm{~cm}$ to the left and right, respectively. This behavior ensures the door opens in front of the user and then closes behind as soon as the user leaves the defined vicinity. To see the user's perspective, see below (Fig. 3)

The user may or needn't be aware of launching a trigger since triggers can be of various types. An example of a trigger is a virtual light switch that actually turns on the lights. Another example is pupil dilation in the user's eye caused by increased cognitive load; this can be used to activate a reduced visual complexity mode of a scene to increase user comfort.

\section{Trigger mechanisms}

The trigger scripts below are those currently available. Note, that the concrete scripts are listed without any environmental context.

- Autonomous trigger. Any trigger can be set with a time delay for execution or reset a number of times (once, $N$ times, indefinitely). An autonomous trigger does this
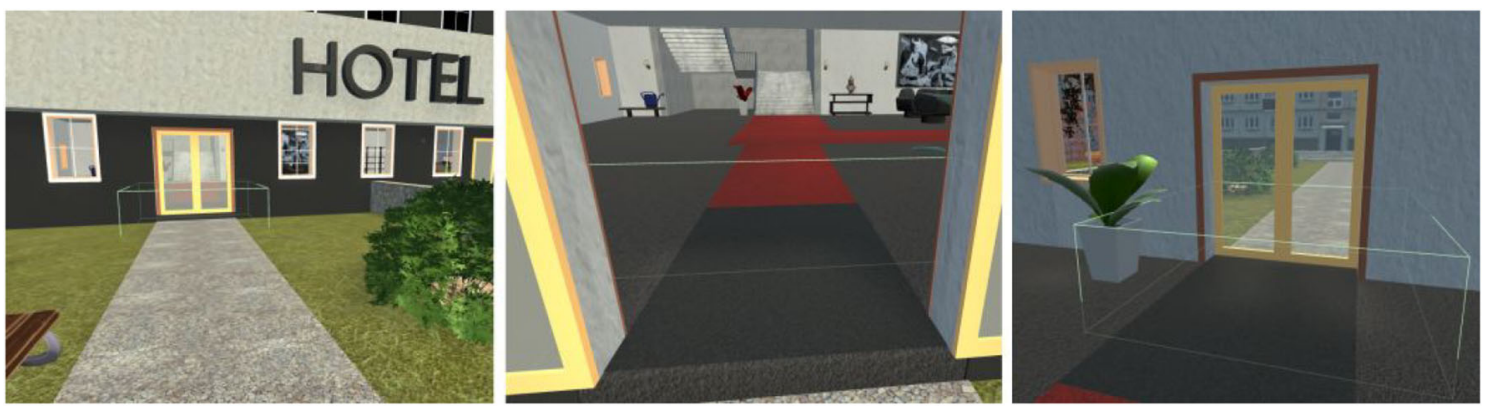

Fig. 3 Behavior example of a two-state trigger. Note. As the user approaches the sliding door, it remains in its default ("off") state. In this particular example, this state is defined as a closed-door system. To emulate the functionality of a real, automated door photosensor, both door wings open when the user enters the defined collider area (highlighted in light green). This activates the trigger and toggles the doors into an "on" (opened) state. When the user leaves the collider area, the trigger again toggles the door system and returns it to the default "off" (closed) state 
independently. For each instance in a specified period (seconds) during the visualization's run-time, the trigger toggles its targeted object(s). In the virtual environment, this usually translates into self-perpetuating or external entity-driven objects, such as traffic lights, animated looping billboards, etc.

- Key trigger. A key trigger defines a list of keys (taken from a generic keyboard or other key-outfitted controller devices), and for each key, a list of objects to trigger. During run-time, the trigger script listens to user key input; if a key existing in its list is pressed, triggering commences.

- Timer trigger. A timer trigger is executed using a key and then follows a predefined sequence of times/objects to trigger. Multi-step object animation or a series of slides in a presentation can be executed using this trigger. This is a key trigger that does not execute a single action but a timed sequence of actions.

- Collider trigger. A triggerable collider is a defined section of virtual space that registers a user's encroachment. If a user enters, exits or remains within the boundaries of this collider for a specified time, the trigger executes. Standard colliders are invisible to the user (undetected trigger type). They may, however, be suggested or implied in the trigger's virtual space environment that an active component is indeed present (e.g., by adding a button, switch, or pressure plate within the collider's range and animating it when a user approaches).

- Collider-key trigger. A combination of the key and collider triggers. Users can only activate a trigger with a keypress if they are present in a certain section of the virtual environment (useful in applying multiple functionalities to the same controller key over multiple virtual areas).

- Distance trigger. This trigger observes the distance between an entity (another user or an (animated) object). If the entity is within a predefined distance interval, the trigger is activated. Multiple intervals can be defined, and if the entity repeatedly moves between them, the script can continue triggering its target objects. In principle, this trigger has similar spatial applications to collider triggers; it can also be used, for example, to implement a custom level of detail (LoD) or scaling on objects (Unity Manual: LoD, 2020).

- Eye-tracking trigger. With integrated VR eye-tracking, a user's gaze can intersect with a virtual object or collider that has an eye-tracking trigger attached to it. The principle resembles a collider trigger: if a gaze enters, exits or fixates for a specified interval, the trigger is activated. Some good practices with eye-tracking should be considered: due to the human eye's rapid searching speed and also the relative inaccuracy of contemporary integrated VR eye-tracker hardware (Clay et al., 2019), some delay in gaze enter and fault tolerance on gaze stay should be specified. However, triggers controlled with eye-tracking are one of the significant advantages offered by Toggle Toolkit since the mechanism could potentially solve the critical question of analyzing eye-tracking data collected in VR.

\section{Toggle script modules}

In contrast to the many trigger scripts, the toolkit's basic toggle script is a universal solution. Using the Unity inspector UI, each instance of the script is attached to the object whose attributes or visuals it will modify.

When toggled, the object transforms into its changed state. When toggled again, it returns to its default state. Such on/off dynamics are the simplest form of the toggle script's functionality and may be compared to the behavior of a light switch or an automated door system (for practical examples, see Figs. 2 and 3; for a schematic depiction, see Fig. 4, left). A togglable object, however, may possess more than two defined states. In such cases, the trigger script executing the toggle script must specify the state that the toggle should switch to (by sending a control number - see Fig. 4, right).

Even in more complex toggle systems (multiple triggers, toggles or toggle states, as described in Chapter 2.2.2), the number of possible states of each object is limited by its attributes and components. Some of these attributes (e.g., position, rotation and scale of an object in virtual space) are universal, but the set of applicable attributes per object is determined by what the specific object allows. If an object does not have a certain component (e.g., when attempting to change the text value of an object that does not have a Unity "Text" component attached), then changing it in the toggle script has no effect.

\section{Toggle functionality}

The sets of attributes currently accessible using a toggle script are listed below. If extra Unity object attributes are required in the future, they may be added to the available toggle script or implemented as a new script, as with components appended to objects in Unity inspector.

- Autonomous behavior. When toggled, the script can be set to execute only once (effectively freezing the object in its changed state). The toggle script can trigger itself as soon as the virtual scene is initiated. It can also delay toggling or toggle itself to its default state once a specified time passes after toggling by a trigger.

- User manipulation (teleportation). An empty object in virtual space with an attached toggle script (with 3D coordinates regardless) can serve as a teleporter. When triggered, it teleports the user to its coordinates. It can also 


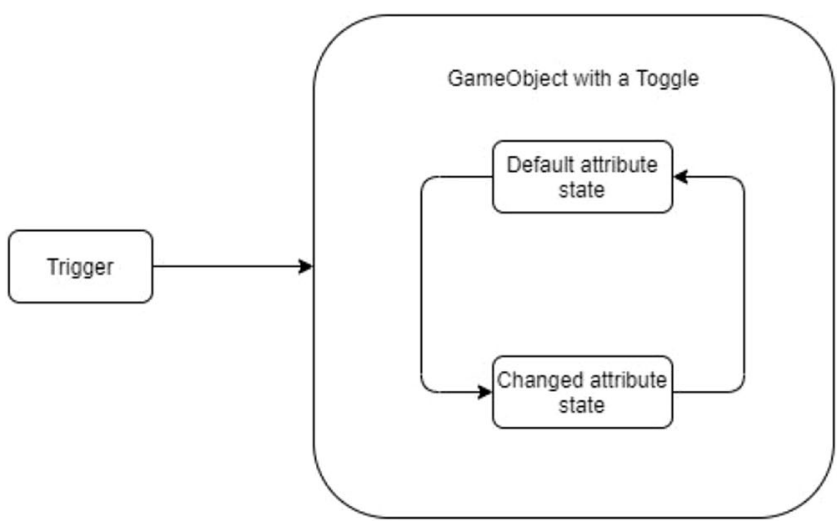

Fig. 4 Overview of simple and complex triggers, with object state transitions. Note. Left - A simple two-state (on/off) toggle. Continuous triggering of the toggle results in switching between the object's default and changed states. Right - A toggle containing more than two

access the user's viewport and change their direction of view (camera angle).

- Light manipulation. If an object has a light component attached to it (any of Unity light), this allows the light to be switched on/off or its intensity and color to be gradually changed.

- Material and text manipulation. As long as the object has a visual representation (a mesh), its material or texture properties can be altered. A simple color change in the object can function as a highlighter, and while this does not change the underlying material, the sudden difference in object tint may be enough to draw the user's attention. Different shaders or Unity materials can also be assigned to the object, changing its visuals more comprehensively.

In some cases, changing an object's text (by using the Unity "Text" component) could be an interchangeable alternative to materials or textures; for example, if the user is exposed to a series of slides.

- Object manipulation. By enabling or disabling its mesh, an object can be visually hidden from a scene. Any object can also be initiated in a scene as a hidden object, only to appear later once it is enabled with a trigger. A set of objects can also be cycled, only one appearing at any given time.

An object in space can be transformed using one or more of the three basic operations: move, scale and rotate. This functionality can transform an object in an instant transition or with a gradual, animated transition. Objects can be transformed relative to their current position/orientation or according to absolute values. A combination of these can produce simple animations. A specialized implementation allows objects to be grabbed/dropped by the user; combined with object physics, this offers a foundation for various complex experimental designs and user task-solving scenarios.

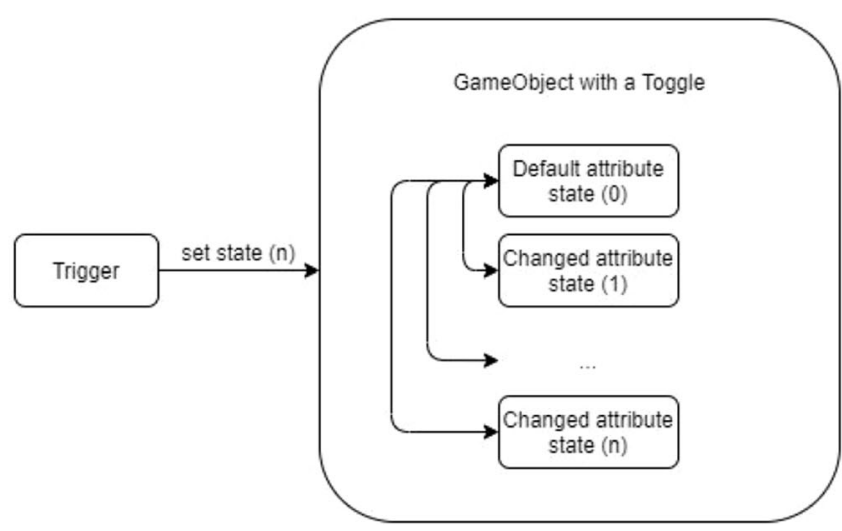

predefined object states. For this to function effectively, the trigger executing the toggle must specify the intended state to switch to (by sending a control number). When activated, the toggle script switches the object to the state specified by the control number

- Audio manipulation. With an AudioSource component attached to an object, audio files can be played. Toggle scripts can store audio files in a list. When an audio file is played, the sound originates from the position (center) of the object. This can add a variety of possibilities to a scene, from environmental sounds to music or narrated instructions.

- External program execution. The script can specify an external program to run, including command-line parameters. For this to work, the external program or its shortcut must be included in one of the operating system's executable directories.

\section{Advanced toggle states}

The basic toggle script is a simple solution: an object is either presented in its default state or a changed state (once triggered). If a trigger toggles this object multiple times, the object would simply cycle between its default and changed states (Figs. 2 and 3). If, however, a more complex state change system is required (e.g., a visual presentation requiring a variety of states to display its slides), a different method must be applied.

A single trigger script can alter multiple togglable objects; similarly, a single togglable object can be triggered with multiple triggers (i.e., an N:N relationship exists between triggers and toggles; for a visual explanation, see Fig. 5, left/right). While linking a single trigger script to an actor's action (e.g., a keypress) may be considered good practice, in some complex cases this may not be desired since the use of multiple triggers features different toggle settings per trigger (multiple triggers used in a manner that complements the dynamic behavior of a targeted object).

Multiple triggers can toggle the same object; this is a feature of our toolkit's design. In addition, each trigger script can 

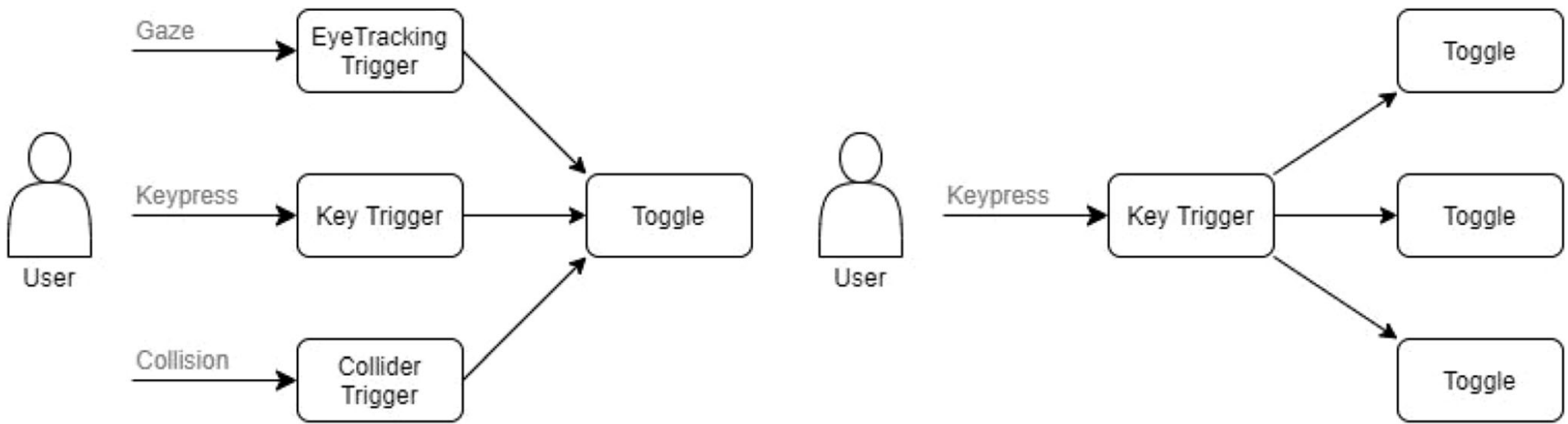

Fig. 5 Relationship between triggers and toggles. Note. Left - Multiple triggers can toggle the same object. Right - A single trigger can toggle multiple objects

also pass a unique control number to its linked toggle script(s) (Fig. 4, right). Since many of the aforementioned toggle functionalities store data in ordered lists (e.g., textures, text fields, audio files), invoking a trigger with a specific control number toggles the object while targeting this number in the list(s). The same can be achieved using a single trigger script, such as a timer trigger, which can be set with multiple triggering actions to trigger the same object with different control numbers over time.

Another use case is in designing experimental scenarios. Some objects may require multiple conditions to be met before they can be triggered. This is solved by introducing an iterator script which serves as an intermediary. The sequence in this setup is: Trigger -Iterator - Toggle. The linked triggers therefore first send their trigger command to an iterator. The conditions that must be satisfied before an iterator passes the trigger to a toggle are defined in the Unity Inspector GUI (e.g., the number of times a trigger must be activated, or a collection of control numbers it receives from triggering). Once these conditions are satisfied, triggering is finally passed to a standard toggle.

Another type of logical dependency is an experimental design that specifies when a second action should follow once a first action completes ( $\rightarrow$ B sequence, i.e., where trigger $\mathrm{A}$ must be activated first, and trigger $B$ remains disabled until trigger $\mathrm{A}$ is activated). This is achieved by attaching the second trigger to a disabled parent GameObject; the first trigger simply enables the disabled parent, and the $\mathrm{A} \rightarrow \mathrm{B}$ sequence may follow.

\section{Data logging modules}

Toggle Toolkit is provided with PathScript, a data logger implementation for experimental applications. When the Unity application initializes, the logger reads the program's settings for the logs it should produce. According to these settings, the logger creates new text file logs with prescribed data formats (CSV or other). The logger has default, predefined log output options, such as user movement or interface use (keyboard $\log$, eye-tracking log, etc.) (Fig. 6). The logger automatically and internally manages these predefined log outputs. It also provides logging functionality to other, external scripts. Thirdparty scripts (e.g., Toggle Toolkit scripts) can access the logger to create their own log files and populate them with their own data (Fig. 7).

Once the experimental application runs, new log entries are written, provided that notable events occur. Some logging is performed periodically (e.g., eye-tracking coordinates), while other logging is per-event only (e.g., key presses, userinitiated triggers).

Understanding the behaviors and events in an interactive toolkit may be a daunting task, especially if a researcher is interested in the interrelations between multiple items. This, however, is not the logger's task. The logger simply outputs logically separated events into separate files or lines. Nevertheless, a researcher may need to know which actor triggered a certain object, which interface was used, or how it affected the virtual environment. This analysis can be done either post hoc, in an external application, or in real-

\begin{tabular}{|l|l|l|l|l|l|l|}
\hline logld & timestamp & hour & min & sec & ms & eventInfo \\
\hline 0 & 1574418894 & 11 & 34 & 54 & 805 & ColliderTrigger point-212-entryPrompt1 triggered Canvas-extra \\
\hline $\mathbf{1}$ & 1574418895 & 11 & 34 & 55 & 462 & ColliderTrigger point-212-entry triggered point-212-entry \\
\hline 2 & 1574418896 & 11 & 34 & 56 & 722 & ColliderTrigger point-212-entryPrompt2 triggered Canvas \\
\hline 3 & 1574418897 & 11 & 34 & 57 & 384 & ColliderTrigger point-212-entryToKitchen triggered point-212-entryToKitchen \\
\hline 4 & 1574418897 & 11 & 34 & 57 & 904 & ColliderTrigger WateringCanPrefab triggered Cube.001 \\
\hline $\mathbf{5}$ & 1574418897 & 11 & 34 & 57 & 909 & ColliderTrigger WateringCanPrefab triggered Canvas \\
\hline $\mathbf{6}$ & 1574418900 & 11 & 35 & 00 & 443 & ColliderTrigger plant2-kitchen triggered Box030 \\
\hline $\mathbf{8}$ & 1574418900 & 11 & 35 & 00 & 444 & ColliderTrigger plant2-kitchen triggered Box030 (1) \\
\hline
\end{tabular}

\begin{tabular}{l|l|}
\hline$\checkmark$ Path Script (Script) \\
Separator Decimal \\
Separator Item & $;$ \\
Allow Custom Logs & $\square$
\end{tabular}

Fig. 6 An example of a standard data output log. Note. An example data output using the internal PathScript logging functionality, showing data for a user's movement through a virtual environment. On the right are the logging settings, set in Unity inspector 


\begin{tabular}{|l|l|l|l|l|l|l|l|l|l|l|l|l|}
\hline logld & timestamp & hour & min & sec & ms & xpos & ypos & zpos & xrot & yrot & zrot & keyPressed \\
\hline 0 & 1574430232 & 14 & 43 & 52 & 57 & 31.90038 & 4.598608 & 31.13223 & 353.2997 & 136.2027 & 0 & up(w) \\
\hline 1 & 1574430232 & 14 & 43 & 52 & 679 & 32.50599 & 4.598608 & 30.55055 & 348.2997 & 133.3027 & 0 & up(w) \\
\hline 2 & 1574430233 & 14 & 43 & 53 & 285 & 33.09337 & 4.598608 & 30.142 & 329.9997 & 137.7027 & 0 & down(s) \\
\hline 3 & 1574430234 & 14 & 43 & 54 & 296 & 32.58818 & 4.598608 & 30.78666 & 5.499656 & 70.40266 & 0 & up(w) \\
\hline 4 & 1574430236 & 14 & 43 & 56 & 705 & 39.34562 & 4.598608 & 33.22945 & 10.89966 & 86.80267 & 0 & right(d) \\
\hline 5 & 1574430237 & 14 & 43 & 57 & 343 & 39.47714 & 4.598608 & 31.5505 & 355.6996 & 176.2027 & 0 & right(d) \\
\hline 6 & 1574430239 & 14 & 43 & 59 & 797 & 38.49111 & 4.587609 & 24.83524 & 4.899662 & 172.7027 & 0 & right(d) \\
\hline 7 & 1574430241 & 14 & 44 & 1 & 342 & 37.7785 & 4.587609 & 21.82908 & 3.699663 & 259.5026 & 0 & left(a) \\
\hline 8 & 1574430245 & 14 & 44 & 5 & 38 & 27.29935 & 4.587609 & 20.00946 & 316.5997 & 286.5026 & 0 & right(d) \\
\hline
\end{tabular}

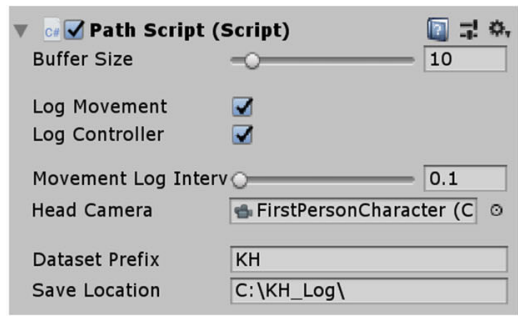

Fig. 7 An example of a custom Toggle Toolkit output log. Note. In this example, the collider trigger execution is logged. At a glance, the structure of the log at left is the same as in the log shown in Fig. 6 because all the logs have a single, predefined data structure, regardless of internal or external origin, as shown in the Unity inspector logging format settings on the right timethrough a custom script that aggregates this type of logging. In any case, the experimenter must decide on the most suitable method. If any data is processed, a specific logic applies to loading or processing different log types separately or cohesively.

External scripts such as those found in the Toggle Toolkit can access and make use of the PathScript logger to produce custom $\log$ files (atomic logs or aggregates of multiple modalities). The existing Toggle Toolkit scripts use PathScript to output their integrated basic logging functionality (e.g., logging trigger/toggle state changes, Fig. 7).

\section{Experimental utility}

The range of options that Toggle Toolkit brings to experimental design is difficult to present in a comprehensive overview. Two case studies are therefore presented in the following sections, with illustrative examples of Toggle Toolkit's utility in experimental research and psychodiagnostics: one depicts a rather simple use of the Toggle Toolkit and the other presents a more complex application. The paper is supplemented with an example of data cleaning and processing session to complete the presentation of how to use Toggle Toolkit.

\section{Complex 3D Scene Processing}

In 2020, our colleagues from Masaryk University conducted an empirical study on processing complex scenes in 1D settings (Čeněk et al., draft). The experiment's design employed eye-tracking, and the experiment's scenery presented to participants consisted of focal objects and a less salient background (a foreground-background task). The experimental hypothesis questioned the intercultural differences in cognitive processing of visual stimuli (Czech and Taiwanese populations).

The aim of this research was to recreate the experiment in immersive 3D and use a VR headset and integrated VR eyetracking to determine whether different sensory input (binocular imaging with depth perception) would create differences to the original findings by Čeněk et al. (2020). We therefore designed a set of complex virtual scenes consisting of a background and two focal objects (Fig. 8).

Toggle Toolkit was used to manage the flow of the experiment. Each of the 30 virtual scenes was separated from the next with an eye-tracking fixation cross. Toggle Toolkit was used to control and time the experiment. The experimenter had several key shortcuts at their disposal to control the experiment. To validate eye-tracker calibration accuracy, users were shown a trial

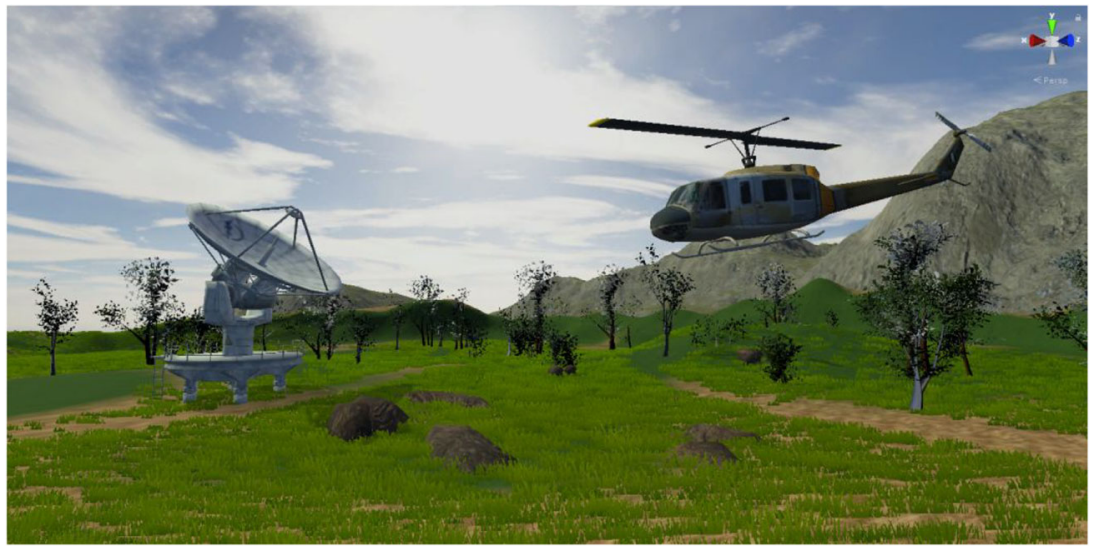

Fig. 8 3D re-creation of an experiment with two focal objects. Note. 3D re-creation of an experiment by Čeněk et al. (2020), with two focal objects, conducted in combination with a VR headset and VR eye-tracking (one of 30 subsequent scenes, this scene displays a radar and helicopter as focal objects, with terrain as a background) 
scene and asked to fixate on certain objects; the exact coordinates of their fixations could then be visualized. With another keypress, the experimenter could then commence the experiment.

\section{Evacuation behavior analysis}

A very practical issue arises in evacuation behavior experiments: no easy way exists to evacuate real buildings without major cost, administration and other investments; in addition, people treat drills differently to real emergencies. We therefore conducted a VR evacuation experiment as a proposed ecologically valid alternative to a real evacuation (Snopková et al., 2020); the interactive segment of the experiment was based on Toggle Toolkit. The entire range of implemented dynamic functionalities is shown in the following video demonstration:

https://rive.google.com/open?id=1 AOhyH9ES08z0JVC gb1QwRxdbtwUHN8Zf

The experiment recreated the layout of an existing hotel in a 3D model (Figs. 2, 3, and 9). Users were outfitted with a VR headset and directed to explore the building. As users navigated, a fire alarm sounded, and users were instructed to exit the building. The experiment was managed by a complex set of spatial triggers, instructions and events, all based on Toggle Toolkit. This allowed providing the users with text instructions while they navigated through the building and give them an interactive task (watering withered plants in one of the hotel's apartments), followed by a fire alarm. Four experimental groups participated in the experiment. Each group was immersed in a slightly different spatial layout of the hotel (differences in corridor width and corridor arrangement). The route inside the hotel was predetermined according to the text instructions, while the subsequent escape route during the fire alarm depended fully on the participants' decisions and strategies. The experiment's hypothesis explored the roles of evacuation signs and spatial layouts on the participants' behavior. Aggregate data for this scenario was logged by placing invisible Toggle Toolkit collider triggers into the environment. Data acquired in this manner later accelerated the process of data analysis.

\section{Discussion}

As illustrated by the schematics and examples in the Software Description chapter and its Toggle Script Modules subchapter, the process of creating a trigger controller logic can be rather complex, especially when multiple triggers are placed in a scene where trigger sequence or dependency is required. When realizing a complex experimental project, we recommend modeling the flow of these scripts and any (in)active objects and toggle states using either the depiction of automata (e.g., UML state machine diagram (Samek, 2008)) or a process modeling tool (jBPM, 2020). This allows the researcher to plan and document their solution before implementation, and if required, review and change it afterwards.

It is worth noting that although only few coding skills are sufficient to use Toggle Toolkit, designing an experiment with this tool provides an opportunity to learn good basics in coding logic since it is necessary to comprehend and well plan trigger-toggle sessions.

Can an experimental design be too complex and involve too many trigger/toggle scripts? Yes, but producing this terminal level of complexity may be beyond most experimental needs. Furthermore, such complexity may be considered from two perspectives: Firstly, it is computational limitations script implementation resulting in costly processor run-time may reduce the fluency of the $3 \mathrm{D}$ experience. Secondly, it is human limitations - it is essential that script dependencies, ineditor clarity, etc., must always be comprehensible to the researcher. Some knowledge of Unity editor is required to design an experiment based on Toggle Toolkit, although this obstacle is not difficult to overcome. Another limitation occurs for experiments that require extreme temporal accuracy,

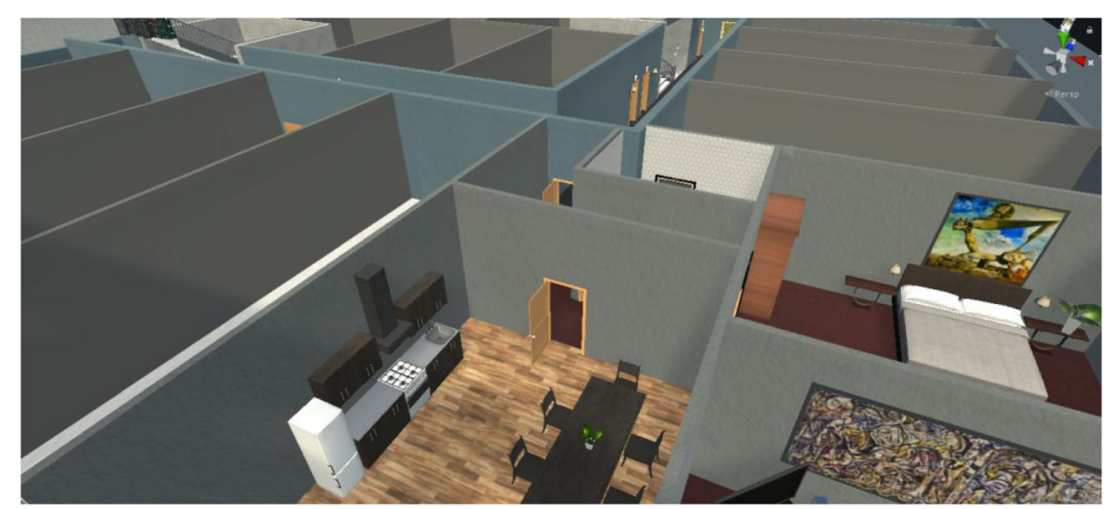

Fig. 9 Overview of the second floor of the hotel in the evacuation experiment. Note. Participants were required to navigate to and subsequently escape from a room on the second floor 
for instance those employing EEG. However, this limit is rooted in the features of the Unity engine, not the toolkit itself. For details on the temporal accuracy, see the supplements including data log examples.

We recommend Toggle Toolkit for use in experimental designs of simple to medium complexity, mainly because of the software's current architecture as described in the present paper. Very complex, specialized implementations with many interconnected functionalities should not be implemented in this manner since a general solution such as Toggle Toolkit will eventually reach the limits of its own software design.

Toggle Toolkit is freely available in a GitHub repository, available at the URL https://github.com/kim-dotcom/ toggleScript. Toggle Toolkit is an open-source product for application in third-party research on the condition that sources are appropriately credited. Toggle Toolkit is also updated, maintained and developed via this GitHub project page. The toolkit will be kept updated for compatibility with new Unity LTS (i.e., long-term support, annual revisions). Thus, the toolkit will be guaranteed to function for at least several years.

Creating custom scripts to extend the scope of the toolkit's functionality is welcomed. Third-party GitHub submissions will be merged on the condition that they follow the aforementioned logic and good practices. If an experimental design with specific needs arises, extensions made to the toolkit for this purpose will also be merged retrospectively. The toolkit still has scope for future development, however, even its current version offers the opportunity to exploit the potential of immersive VR in research on human behavior with little effort and further training on the side of researchers.

Supplementary Information The online version contains supplementary material available at https://doi.org/10.3758/s13428-020-01510-4.

Acknowledgements This publication was supported by the Czech Science Foundation (GC19-09265J: The influence of socio-cultural factors and writing system on perception and cognition of complex visual stimuli).

Toggle Toolkit programming, debugging and pilot testing of experiments was made available by the infrastructure of Virtual Geographic Environments Lab, Department of Geography, Faculty of Science, Masaryk University.

Experiment preparation and data collection were aided by the infrastructure of the Grey Lab, Department of Psychology, Faculty of Arts, Masaryk University.

This work was supported by the research infrastructure HUME Lab Experimental Humanities Laboratory, Faculty of Arts, Masaryk University.

\section{References}

2D Game Kit Reference Guide (2017). Retrieved from https://learn.unity. $\mathrm{com} /$ tutorial/2d-game-kit-reference-guide
Alcañiz, M., Rey, B., Tembl, J., Parkhutik, V. (2009). A Neuroscience Approach to Virtual Reality Experience Using Transcranial Doppler Monitoring. Presence: Virtual and Augmented Reality 18(2), 97111. https://doi.org/10.1162/pres.18.2.97

Baron, D. (2019). Hands-on game development patterns with Unity 2019: create engaging games by using industry-standard design patterns with C\#. Birmingham, UK: Packt Publishing.

Brookes, J., Warburton, M., Alghadier, M., Mon-Williams, M., \& Mushtaq, F. (2019). Studying human behavior with virtual reality: The Unity Experiment Framework. Behavior Research Methods. https://doi.org/10.3758/s13428-019-01242-0

Caputo, F. M., Emporio, M., \& Giachetti, A. (2018). The Smart Pin: An effective tool for object manipulation in immersive virtual reality environments. Computers \& Graphics, 74, 225-233. https://doi. org/10.1016/j.cag.2018.05.019

Čeněk, J., Tsai, JL., Šašinka, Č. (2020). Cultural Variations in Global and Local Attention and Eye-Movement Patterns during the Perception of Complex Visual Scenes: Comparison of Czech and Taiwanese University Students. PLoS ONE 15(11). https://doi.org/ 10.1371/journal.pone.0242501

Clay, V., König, P., \& König, S. (2019). Eye Tracking in Virtual Reality. Journal of Eye Movement Research, 12(1), 1-18. https://doi-org. ezproxy.muni.cz/10.16910/jemr.12.1.3

Cornelissen, F.W.; Peters, E.M.; Palmer, J. The EyeLink Toolbox: Eye tracking with MATLAB and the Psychophysics Toolbox. Behav. Res. Method. Instrum. Comput. 2002, 34, 613-671.

Cuervo, E., Chintalapudi, K., \& Kotaru, M. (2018). Creating the Perfect Illusion. Proceedings of the 19th International Workshop on Mobile Computing Systems \& Applications - HotMobile 18. https://doi.org/ $10.1145 / 3177102.3177115$

D., R., (2015, September 29). The maturity of visual programming. Retrieved from https://craft.ai/blog/the-maturity-of-visualprogramming

Diersch, N., \& Wolbers, T. (2019). The potential of virtual reality for spatial navigation research across the adult lifespan. The Journal of Experimental Biology, 222(Suppl 1). https://doi.org/10.1242/jeb. 187252

Fuad, M. (2019, December 17). XR Interaction Toolkit Preview Package is here - Unity Technologies Blog. Unity Technologies Blog. Retrieved from https://blogs.unity3d.com/2019/12/17/xrinteraction-toolkit-preview-package-is-here/

Gibson, J. J. (1977). The theory of affordances. Hilldale, USA, 1(2).

Gould, N. F., Holmes, M. K., Fantie, B. D., Luckenbaugh, D. A., Pine, D. S., Gould, T. D., \& Zarate, C. A. (2007). Performance on a virtual reality spatial memory navigation task in depressed patients. American Journal of Psychiatry, 164(3), 516-519. https://doi.org/ 10.1176/ajp.2007.164.3.516.

Grübel, J., Thrash, T., Hölscher, C., \& Schinazi, V. R. (2017). Evaluation of a conceptual framework for predicting navigation performance in virtual reality. PLoS one, 12(9), e0184682. doi:https://doi.org/10. 1371/journal.pone. 0184682

jBPM (2020). Retrieved from https://www.jbpm.org/

Kovalčík, V., Chmelík, J., Bezděka, M., \& Sochor, J. (2012). Virtual reality system as a tool for education. In Václav Skala (Ed.), paper presented at the WSCG Poster proceedings (15-18). Plzen: Union Agency.

Kubíček, P., Šašinka, Č., Stachoň, Z., Herman, L., Juřík, V., Urbánek, T., \& Chmelík, J. (2017). Identification of altitude profiles in 3D geovisualizations: the role of interaction and spatial abilities. International Journal of Digital Earth, 12(2), 156-172. https://doi. org/10.1080/17538947.2017.1382581

Loomis, J. M., Blascovich, J. J., \& Beall, A. C. (1999). Immersive virtual environment technology as a basic research tool in psychology. Behavior Research Methods, Instruments, \& Computers, 31(4), 557-564. https://doi.org/10.3758/bf03200735 
Mathôt, S.; Schreij, D.; Theeuwes, J. OpenSesame: An open-source, graphical experiment builder for the social sciences. Behavior Research Method. 2012, 44, 314-324.

Moghadam, K. R., Banigan, C., \& Ragan, E. D. (2018). Scene Transitions and Teleportation in Virtual Reality and the Implications for Spatial Awareness and Sickness. IEEE Transactions on Visualization and Computer Graphics, 1-1. https://doi.org/10.1109/tvcg.2018.2884468

Neuro behavioral systems, Inc. Available online: www.neurobs.com (accessed on 24 February 2017).

Paradigm. Available online: http://www.paradigmexperiments.com (accessed on 24 February 2017).

Parong, J., \& Mayer, R. E. (2018). Learning science in immersive virtual reality. Journal of Educational Psychology, 110(6), 785-797. https://doi.org/10.1037/edu0000241

Samek, M. (2008). Practical UML Statecharts in C/C++, Second Edition: Event-Driven Programming for Embedded Systems. London, UK: Routledge.

Sewell, B. (2015). Blueprints visual scripting for unreal engine: build professional 3D games with Unreal Engine 4s Visual scripting system. Birmingham: Packt Publishing.

Šašinka, Č., Morong, K., Stachoň, Z. (2017). The Hypothesis Platform: An Online Tool for Experimental Research into Work with Maps and Behavior in Electronic Environments. International Journal of Geo-Information, 6(12). https://doi.org/10.3390/ijgi6120407

Singer, G. (2013). The History of the Modern Graphics Processor. Retrieved from: http://www.techspot.com/article/650-history-ofthe-gpu/
Smith, S. P., \& Trenholme, D. (2009). Rapid prototyping a virtual fire drill environment using computer game technology. Fire Safety Journal, 44(4), 559-569. https://doi.org/10.1016/j.firesaf.2008.11. 004

Snopková, D., Ugwitz, P., Stachoň, Z., Hladík, J., Kvarda, O., \& Kubíček, P. (2020). Can increased visual accessibility and signage suppress retracing evacuation tendencies in unfamiliar buildings? A Virtual reality game-based experiment. Manuscript submitted for publication.

Unity Manual: Components (2020). Retrieved from: https://docs.unity3d. com/Manual/Components.html

Unity Manual: LoD (2020). Retrieved from https://docs.unity3d.com/ Manual/LevelOfDetail.html

Unity Scripting Reference (2020). Retrieved from: https://docs.unity3d. com/ScriptReference/

Vasser, M., Kängsepp, M., Magomedkerimov, M., Kilvits, K., Stafinjak, V., Kivisik, T., Vicente, R., \& Aru, J. (2017). VREX: an opensource toolbox for creating 3D virtual reality experiments. BMC Psychology. https://doi.org/10.1186/s40359-017-0173-4

Vizard. Virtual Reality software for researchers (2020). Retrieved from https://worldviz.com/vizard-virtual-reality-software

Publisher's note Springer Nature remains neutral with regard to jurisdictional claims in published maps and institutional affiliations. 